\title{
Ir40a neurons are not DEET detectors
}

$\mathrm{N}, \mathrm{N}$-Diethyl-meta-toluamide (DEET) is the most widely used insect repellent, but it requires repeated application at high, potentially harmful, concentrations, which is prohibitively impractical and costly in the countries suffering most from insect vector-borne diseases ${ }^{1}$; understanding DEET's mode of action might help identify improved alternatives. Kain et al. ${ }^{2}$ characterized ionotropic receptor 40a (Ir40a)-expressing olfactory sensory neurons (OSNs)-located in the sacculus of the Drosophila antenna ${ }^{3}$ - as comprising a key pathway mediating aversion to both DEET and chemoinformatically predicted 'DEET-like' compounds, using calcium imaging, a transcription-based neural activity reporter, Ir40a RNA interference and behavioural experiments. We are unable to reproduce evidence for physiological activation of Ir40a OSNs by these repellents, and find that Ir40a mutant flies avoid DEET. These results call into question the importance of this sensory pathway in DEET detection. There is a Retraction accompanying this Brief Communication Arising by Kain, P. et al. Nature 534, http://dx.doi.org/10.1038/ nature18613 (2016).

Ir40a-expressing OSN dendrites innervate sacculus chambers I and II (Fig. 1a), and their axons project to the 'arm' and 'column' in the antennal lobe (Fig. 1b) ${ }^{4}$. Using a calcium imaging-based screen for natural stimuli capable of activating these neurons (Extended Data Fig. 1), we identified ammonia as an agonist that evokes dose-dependent responses in the axon termini of all Ir40a neurons (Fig. 1c, d). This chemical specificity is consistent with the phylogenetic grouping of Ir40a with other members of the IR family of variant ionotropic glutamate receptors that detect amines ${ }^{4}$. Surprisingly, we were unable to reproduce the reported activation of Ir40a neurons by DEET or DEETlike compounds (butyl anthranilate, ethyl anthranilate and methyl $N, N$-dimethylanthranilate) ${ }^{2}$ (Fig. 1e). To rule out insufficient stimulus concentration as a cause for the lack of responses, we presented these chemicals directly to the antenna by manual puffing from a syringe, as described ${ }^{2}$. Using this method, we detected strong responses to ammonia, but not to DEET or DEET-like chemicals in Ir40a neurons (Fig. 1f). We also visualized calcium levels in Ir40a OSN soma, as described ${ }^{2}$, but this again revealed responses to ammonia but not DEET or DEET-like compounds (Fig. $1 \mathrm{~g}$ and Supplementary Videos 1-8).

Ir40a neurons were initially implicated as DEET sensors through their activation of a transcriptional reporter of neural activity (calciumdependent nuclear import of LexA, CaLexA; ref. 5) - expressed in all antennal neurons with the driver elav-Gal4-upon long-term DEET exposure $^{2}$. The reliability of this reporter is, however, unclear, as less than half ( 2 to 3 out of 6 ) presumed Ir40a OSNs in chamber I and no chamber II OSNs were described to exhibit DEET-evoked CaLexA activity $^{2}$. This seems not to be due to insufficient DEET, as exposure to $10 \%$ or $100 \%$ DEET led to similar CaLexA reporter results ${ }^{2}$. We therefore repeated these experiments and found, unexpectedly, that among the entire population of antennal neurons, chamber I neurons are particularly susceptible to background CaLexA signals, which were equivalent in flies exposed to no odour, DEET or ammonia (Fig. 1h). Such signals might be due to high basal calcium levels and/or basal nuclear translocation of abundantly expressed CaLexA reporter in these cells; regardless, our observations indicate that, under these experimental conditions, CaLexA cannot be used to report on stimulusevoked activity of sacculus chamber I neurons.

To assess the function of Ir40a, we generated Ir40a antibodies and, by CRISPR/Cas9 genome editing, an Ir40a mutant (Extended Data Fig. 2a, b). In wild-type flies, Ir40a co-localizes with the co-receptor Ir 25a (ref. 6) in OSN soma and dendrites, suggesting that these function together in sensory detection; in Ir40a mutants, anti-Ir40a immunoreactivity is absent (Extended Data Fig. 2b), confirming this allele to be a protein null. Consistent with these observations, physiological responses of Ir40a OSNs to ammonia are abolished in both Ir40a and Ir 25 a mutant animals (Extended Data Fig. 2c).

We tested behavioural responses of Ir40a and Ir $25 a$ mutants in a DEET olfactory aversion assay (Fig. 1i-j). Both mutants avoid DEET as robustly as wild-type controls; in contrast, mutants for the odorant receptor co-receptor Orco no longer avoid DEET (Fig. 1j), confirming previous data in both Drosophila ${ }^{7}$ and mosquitoes ${ }^{8,9}$ that olfactory detection of DEET is OR-dependent.

In summary, our data fail to reproduce evidence for physiological activation of Ir40a neurons by DEET, or to demonstrate a genetic requirement for either Ir40a or the co-receptor Ir25a in behavioural avoidance of this repellent. We propose that this sensory pathway is not relevant for DEET detection; future work will determine its role in behavioural responses to ammonia, an olfactory cue of ecological importance for many insects ${ }^{10,11}$.

\section{Methods}

Most experiments used published reagents and approaches as follows, with minor modifications: antenna and antennal lobe histology ${ }^{4,12}$; antenna and antennal lobe calcium imaging ${ }^{13,14}$; manual odour delivery ${ }^{2}$; CaLexA assay ${ }^{2,5}$; CRISPR/Cas9 genome editing ${ }^{15}$; behavioural assays ${ }^{2,7}$. Ir40a guinea pig polyclonal antibodies were raised against the peptide KERNVYLDDFLQRLHRSNYK. New transgenes: to generate UAS-Ir $40 a$ we inserted the Ir $40 a$ open reading frame into $p U A S T$ attB, and to generate Ir40a-LexA we inserted the Ir40a promoter ${ }^{4}$ into $p L e x A: V P 16$ SV40tail attB. Full methods are provided in the Supplementary Information.

Ana F. Silbering ${ }^{1}$, Rati Bell ${ }^{1}$, Daniel Münch ${ }^{2}$, Steeve Cruchet $^{1}$, Carolina Gomez-Diaz ${ }^{1} \dagger$, Thomas Laudes ${ }^{2}$, C. Giovanni Galizia ${ }^{2}$ \& Richard Benton ${ }^{1}$

${ }^{1}$ Center for Integrative Genomics, Faculty of Biology and Medicine, University of Lausanne, Lausanne, CH-1015, Switzerland.

2Department of Biology, University of Konstanz, 78457, Konstanz, Germany.

email: Richard.Benton@unil.ch

$\dagger$ Present address: Department of Biology, University of Konstanz, 78457, Konstanz, Germany.

1. Leal, W. S. The enigmatic reception of DEET—-the gold standard of insect repellents. Curr. Opin. Insect Sci. 6, 93-98 (2014).

2. Kain, P. et al. Odour receptors and neurons for DEET and new insect repellents. Nature 502, 507-512 (2013).

3. Benton, R., Vannice, K. S., Gomez-Diaz, C. \& Vosshall, L. B. Variant ionotropic glutamate receptors as chemosensory receptors in Drosophila. Cell 136, 149-162 (2009).

4. Silbering, A. F. et al. Complementary function and integrated wiring of the evolutionarily distinct Drosophila olfactory subsystems. J. Neurosci. 31, 13357-13375 (2011). 
a

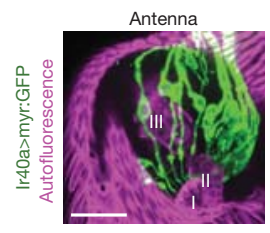

b

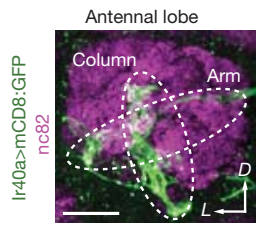

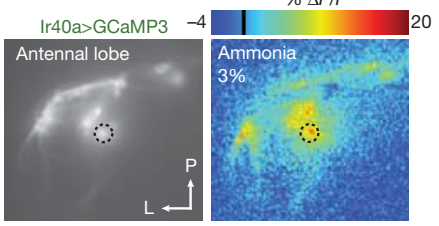

d
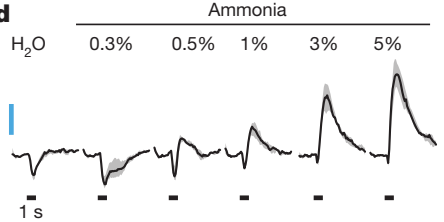

e Antennal lobe, automated stimulation

1

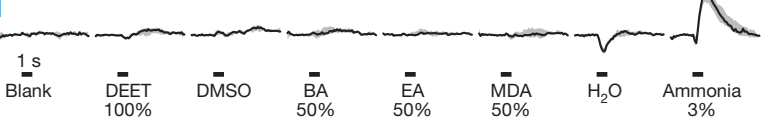

f Antennal lobe, manual stimulation

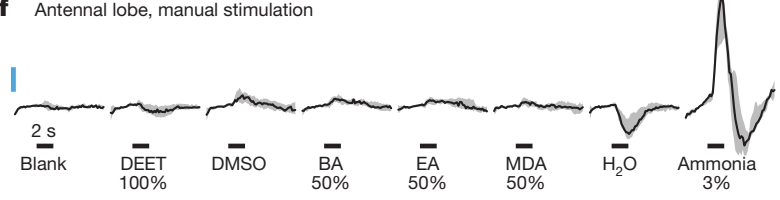

Figure 1 | Ir40a neurons are activated by ammonia but not DEET, and avoidance of DEET does not require Ir40a or the co-receptor Ir25a.

a, Confocal image of a whole-mount antenna of an Ir40a-Gal4/10 $\times$ UASmyr:GFP fly stained for anti-green fluorescent protein (GFP) (green), revealing innervation of Ir40a neurons in sacculus chambers I and II. Cuticular autofluorescence (magenta) provides anatomical landmarks. Ir40a $>$ myr:GFP indicates that a membrane-targeted myristoylated GFP reporter is expressed under the control of Ir40a-Gal4. The scale bar is $20 \mu \mathrm{m}$. b, Confocal stack of one antennal lobe in a whole-mount brain of an Ir40a-Gal4/+;UAS-mCD8:GFP/+ fly stained for anti-GFP (green) and the synaptic neuropil marker nc82 (magenta), revealing projections of Ir40a neurons to the column and arm glomeruli. Ir40a > mCD8:GFP indicates that a membrane-targeted mCD8:GFP fusion protein reporter is expressed under the control of Ir40a-Gal4. The scale bar is $20 \mu \mathrm{m}$. c, Left, wide-field fluorescence image of the right antennal lobe of an Ir40a-Gal4;UAS-GCaMP3 fly, highlighting the region of interest (marked by a dashed circle) used for quantification of stimulus-evoked calcium responses in axon termini (note the antennal lobe has a different orientation from that of $\mathbf{b}$ ). Right, colour-coded image of the response to ammonia (Chemical Abstracts Service (CAS) 1336-21-6) (3\% v/v in $\left.\mathrm{H}_{2} \mathrm{O}\right)$. The colour scale indicates the percentage fluorescence change relative to the baseline $(\% \Delta F / F)$ the image corresponds to the $\Delta F / F$ of the peak of the response. $\mathbf{d}$, Traces of the median response of Ir40a neurons ( $n=6$ flies) to the indicated stimuli $(20 \mu$ l on a Sugi pad in $2 \mathrm{ml}$ syringe; automated delivery ${ }^{13}$ indicated by black horizontal bars). The grey surface indicates the range between the first and third quartiles of the distribution, here and in panels $\mathbf{e}-\mathbf{g}$. The fluorescence decrease in response to the $\mathrm{H}_{2} \mathrm{O}$ solvent is overcome by increasing positive responses to higher concentrations of ammonia. The vertical blue scale bar, here and in panels e-g, indicates $5 \% \Delta F / F$. e, Calcium responses of Ir40a neurons to the indicated stimuli measured as in d. DEET (CAS 134-62-3) was undiluted; 'DEET-like' compounds butyl anthranilate (BA; CAS 7756-96-9), ethyl anthranilate (EA; CAS 87-25-2) and methyl $N, N$-dimethylanthranilate (MDA; CAS 10072-05-6) were dissolved in dimethylsulfoxide (DMSO; CAS 67-68-5) ( $n=5$ flies for blank, 6 flies for DMSO, butyl anthranilate, ethyl anthranilate and methyl $\mathrm{N}, \mathrm{N}$-dimethylanthranilate and 11 flies for DEET, $\mathrm{H}_{2} \mathrm{O}$ and ammonia). f, Calcium responses of Ir40a neurons to the indicated stimuli delivered manually ${ }^{2}$ to the antenna $(n=9$ flies for ammonia and 11 flies for all other stimuli). g, Calcium responses of Ir40a OSN soma (imaged in antennae by confocal microscopy ${ }^{14}$ ) to the indicated stimuli delivered
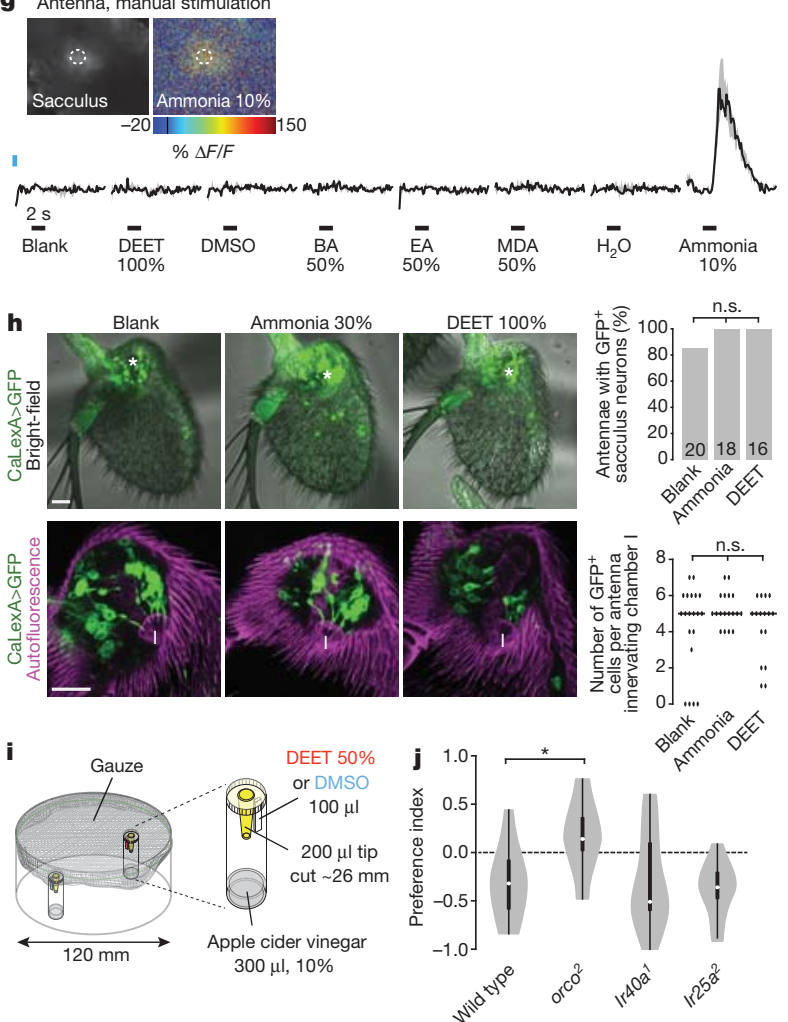

manually ${ }^{2}$ ( $n=5$ flies). The inset panels show raw GCaMP3 fluorescence in sacculus neurons (region of interest marked by a dashed circle) and a colour-coded image of the peak of the response to ammonia. $\mathbf{h}$, Top: GFP reporter signal (anti-GFP, green) in antennae of pan-neural CaLexA flies (elav-Gal4/LexAop-CD8:GFP-2A-CD8:GFP;UAS-mLexA:VP16:NFAT,LexAop$C D 2: G F P /+$ ) (ref. 5) exposed to the indicated stimuli (blank, $20 \mu \mathrm{l}$ ammonia or $100 \mu \mathrm{l}$ DEET on a Sugi pad), overlaid on a bright-field image that shows the anatomical landmarks (the asterisk marks the sacculus). Quantification is shown on the right ( $n$ is shown in each bar). There is no significant difference in the number of antennae with reporter signal around the sacculus across stimuli ( $\chi^{2}$ test, $P>0.05$ ). The scale bar is $20 \mu \mathrm{m}$. Bottom: GFP reporter signal (anti-GFP, green) in high-magnification images of the sacculus in CaLexA flies exposed to the indicated stimuli. Cuticular autofluorescence (magenta) provides anatomical landmarks (chamber I is marked with ' $\mathrm{I}$ '). The scale bar is $20 \mu \mathrm{m}$. Quantification of GFP-positive sacculus chamber I neurons is shown on the right; the horizontal line represents the median number of labelled neurons for each treatment. There is no significant difference in the number of chamber I neurons with reporter signal between stimuli (Wilcoxon test all pairwise comparisons, $P$-value corrected for multiple testing using Benjamini and Hochberg's method). n.s., not significant. i, Schematic of the behavioural assay: in a plastic arena, flies ( $\sim 25$ females, $2-5$ days post-eclosion) can choose, over $24 \mathrm{~h}$ at $25^{\circ} \mathrm{C}$ under illumination, to enter plastic traps containing $300 \mu \mathrm{l}$ apple cider vinegar, with either $100 \mu$ l of $50 \%$ DEET (in DMSO) or DMSO alone spotted on a Sugi pad positioned near the trap entrance ensuring that flies cannot contact the Sugi pad before trap entry. $\mathbf{j}$, The preference index of the indicated genotypes was calculated as: (number of flies in DEET trap minus number of flies in control trap)/(number of trapped flies plus number of non-trapped flies); negative values indicate DEET avoidance. $n=20$ traps for Ir $25 a^{2}$ and $o r c o^{2}$ and 22 traps for $\operatorname{Ir} 40 a^{1}$ (superscripts 1 and 2 indicate mutant alleles) and wild type $\left(w^{1118}\right)$ (about two traps per genotype per day over ten experimental days); average trapping rate was $>90 \%$ for all genotypes in all experiments. The white dot indicates the median of the distribution, the thick black line the range between the first and third quartile of the distribution and the grey area shows a rotated kernel density plot on each side of the boxplot. Mutant genotypes were compared to the $w^{1118}$ wild type (Wilcoxon test corrected for multiple testing using Benjamini and Hochberg's method; $* P<0.05)$. 
5. Masuyama, K., Zhang, Y., Rao, Y. \& Wang, J. W. Mapping neural circuits with activity-dependent nuclear import of a transcription factor. J. Neurogenet. 26, 89-102 (2012).

6. Abuin, L. et al. Functional architecture of olfactory ionotropic glutamate receptors. Neuron $69,44-60$ (2011).

7. Ditzen, M., Pellegrino, M. \& Vosshall, L. B. Insect odorant receptors are molecular targets of the insect repellent DEET. Science 319, 1838-1842 (2008).

8. Xu, P., Choo, Y. M., De La Rosa, A. \& Leal, W. S. Mosquito odorant receptor for DEET and methyl jasmonate. Proc. Natl Acad. Sci. USA 111, 16592-16597 (2014).

9. DeGennaro, M. et al. orco mutant mosquitoes lose strong preference for humans and are not repelled by volatile DEET. Nature 498, 487-491 (2013).

10. Geier, M., Bosch, O. J. \& Boeckh, J. Ammonia as an attractive component of host odour for the yellow fever mosquito, Aedes aegypti. Chem. Senses 24, 647-653 (1999).

11. Min, S., Ai, M., Shin, S. A. \& Suh, G. S. Dedicated olfactory neurons mediating attraction behavior to ammonia and amines in Drosophila. Proc. Natl Acad. Sci. USA 110, E1321-E1329 (2013).

12. Saina, M. \& Benton, R. Visualizing olfactory receptor expression and localization in Drosophila. Methods Mol. Biol. 1003, 211-228 (2013).
13. Silbering, A. F., Bell, R., Galizia, C. G. \& Benton, R. Calcium imaging of odor-evoked responses in the Drosophila antennal lobe. J. Vis. Exp. 61, 2976 (2012).

14. Strauch, M. et al. More than apples and oranges-detecting cancer with a fruit fly's antenna. Sci. Rep. 4, 3576 (2014).

15. Bassett, A. R. \& Liu, J. L. CRISPR/Cas9 and genome editing in Drosophila. J. Genet. Genom. 41, 7-19 (2014) 